\title{
PTEN status switches cell fate between premature senescence and apoptosis in glioma exposed to ionizing radiation
}

\author{
J-J Lee ${ }^{1}$, BC Kim ${ }^{1}$, M-J Park ${ }^{1}$, Y-S Lee ${ }^{2}$, Y-N Kim ${ }^{3}$, BL Lee ${ }^{4}$ and J-S Lee ${ }^{*, 1}$
}

Loss of the tumor suppressor phosphatase and tensin homolog (PTEN) has frequently been observed in human gliomas, conferring AKT activation and resistance to ionizing radiation (IR) and drug treatments. Recent reports have shown that PTEN loss or AKT activation induces premature senescence, but many details regarding this effect remain obscure. In this study, we tested whether the status of PTEN determined fate of the cell by examining PTEN-deficient U87, U251, and U373, and PTEN-proficient LN18 and LN428 glioma cells after exposure to IR. These cells exhibited different cellular responses, senescence or apoptosis, depending on the PTEN status. We further observed that PTEN-deficient U87 cells with high levels of both AKT activation and intracellular reactive oxygen species (ROS) underwent senescence, whereas PTEN-proficient LN18 cells entered apoptosis. ROS were indispensable for inducing senescence in PTEN-deficient cells, but not for apoptosis in PTENproficient cells. Furthermore, transfection with wild-type (wt) PTEN or AKT small interfering RNA induced a change from premature senescence to apoptosis and depletion of p53 or p21 prevented IR-induced premature senescence in U87 cells. Our data indicate that PTEN acts as a pivotal determinant of cell fate, regarding senescence and apoptosis in IR-exposed glioma cells. We conclude that premature senescence could have a compensatory role for apoptosis in the absence of the tumor suppressor PTEN through the AKT/ROS/p53/p21 signaling pathway.

Cell Death and Differentiation (2011) 18, 666-677; doi:10.1038/cdd.2010.139; published online 12 November 2010

Gliomas are the most common type of brain tumor, and grade IV glioblastomas are almost universally fatal. Treatment of glioblastomas typically involves surgical resection in combination with radiation and alkylating agent-based adjuvant chemotherapy. However, even in instances in which complete surgical resection of theses tumors is possible, the tumor generally recurs within a year, regardless of its initial response to treatment. ${ }^{1}$ Oncogenes (EGF, PDGF, and their receptors) and tumor suppressor genes $\left(p 16^{I N K 4 a}, p 14^{A R F}, P T E N, R B 1\right.$, and TP53) are involved in the evolution of glioblastomas, ${ }^{2}$ and activation of the phosphatidylinositol 3-kinase (PI3K) signaling pathway has a crucial role in their development. ${ }^{3}$ The tumor suppressor gene PTEN encodes a lipid phosphatase that counteracts the effect of PI3K signaling, thereby negatively controlling the activation of this pathway. Tumor suppressor PTEN is mutationally and transcriptionally inactivated in many different tumor types, including glioblastoma. ${ }^{3}$ A central node in signaling events downstream of PI3K is controlled by the serine-threonine kinase AKT. Therefore, AKT is activated by PI3K, which generates phosphatidylinositol 3, 4, 5-trisphosphate, and is negatively regulated by phospholipid phosphatases PTEN. ${ }^{4}$ Hyperactivated AKT provides protection from apoptosis and promotes uncontrolled cell cycle progression. ${ }^{5}$ However, it has recently been shown that AKT activity increases with cellular senescence, and that inhibition of AKT extends the lifespan of primary cultured human endothelial cells. ${ }^{6}$

Cellular senescence is an extremely stable form of cell cycle arrest, which is activated in response to stress, including oncogenic signaling and telomere shortening. ${ }^{7}$ The initial description of cellular senescence by Hayflick and Moorehead was based on the endurable analysis of normal human cells grown in vitro. ${ }^{8}$ In contrast to cancer cells, they observed that normal cells had a finite proliferative capacity that ended in stable and long-term cell cycle arrest. Considering that neoplastic transformation involves events that inhibit the program of senescence, tumor cells were believed to have lost the ability to senescence. However, recent data has shown that tumor cells can be readily induced to undergo senescence by genetic manipulation, or by treatment with chemotherapeutic drugs, radiation, or differentiation agents. ${ }^{9}$ The importance of cellular senescence is increasingly being recognized as a tumor suppression mechanism. ${ }^{10,11}$

Here, we show that after ionizing radiation (IR) exposure, PTEN acts as a critical determinant of cell fate between senescence and apoptosis in the glioma cell lines U87, U251, U373, LN18, and LN428. The U87, U252, and U373 cells underwent senescence by $\mathrm{p} 21$ induction because of mutation

\footnotetext{
${ }^{1}$ Division of Radiation Cancer Research, Korea Institute of Radiological and Medical Sciences, Seoul, Korea; ${ }^{2}$ College of Pharmacy \& Division of Life Science and Pharmaceuticals, Ewha Womans University, Seoul, Korea; ${ }^{3}$ Pediatric Oncology Division, National Cancer Center, Gyeonggi-do, Korea and ${ }^{4}$ Department of Anatomy, Seoul National University College of Medicine, Seoul, Korea

${ }^{*}$ Corresponding author: J-S Lee, Division of Radiation Cancer Research, Korea Institute of Radiological and Medical Sciences, Seoul 139-706, Korea.

Tel: + 822970 1388; Fax: + 822970 1388; E-mail: jaeslee@kcch.re.kr

Keywords: premature senescence; apoptosis; PTEN; glioma

Abbreviations: BrdU, bromodeoxyuridine; $\mathrm{H}_{2} \mathrm{O}_{2}$, hydrogen peroxide; NAC, $N$-acetyl-I-cysteine; ROS, reactive oxygen species; RT, radiotherapy; SA- $\beta$-Gal, senescence-associated $\beta$-galactosidase; siRNA, small interfering RNA; wt, wild-type

Received 10.5.10; revised 20.9.10; accepted 30.9.10; Edited by JA Cidlowski; published online 12.11.10
} 
or deficiency of PTEN. However, LN428 and LN18 cells, which express wild-type (wt) PTEN, displayed apoptotic, as opposed to senescent, phenotypes after IR exposure. Further examinations suggest that premature senescence could be an alternative mechanism to prevent aberrant cell proliferation, instead of apoptosis, in the absence of the tumor suppressor PTEN.

\section{Results}

PTEN status dictates premature senescent and apoptotic phenotypes in glioma cells following IR exposure. To test whether the cellular response of glioma to IR is associated with PTEN status, we exposed several glioma cell lines, including PTEN-deficient U87, U251, and U373 and the PTEN-proficient LN18 and LN428 cells to various doses of IR. We first examined relative cell numbers and cellular morphologies, and observed that all cell types had decreased cell numbers in a dose-dependent manner following IR (Figure 1a). Furthermore, microscopic analyses indicated that U87, U251, and U373 cells were positive for senescence-associated $\beta$-galactosidase (SA- $\beta$-Gal), a hallmark of senescence, and for senescent morphology (large flattened shape) (Figure 1b). In contrast, LN18 and LN428 cells became susceptible to trypan blue staining, and displayed Annexin $\mathrm{V}$ positivity, in a dose-dependent manner (Figure 1c). Cyclin-dependent kinase inhibitor p21, one of senescence markers, was dramatically increased in PTEN-deficient U87, U251, and U373 cells, but not in PTEN-proficient LN18 and LN428 cells, and cleaved poly (ADP-ribose) polymerase (PARP), which is an indicator of the biochemical changes due to caspase activation during apoptosis was detected only in LN18 and LN428 cells
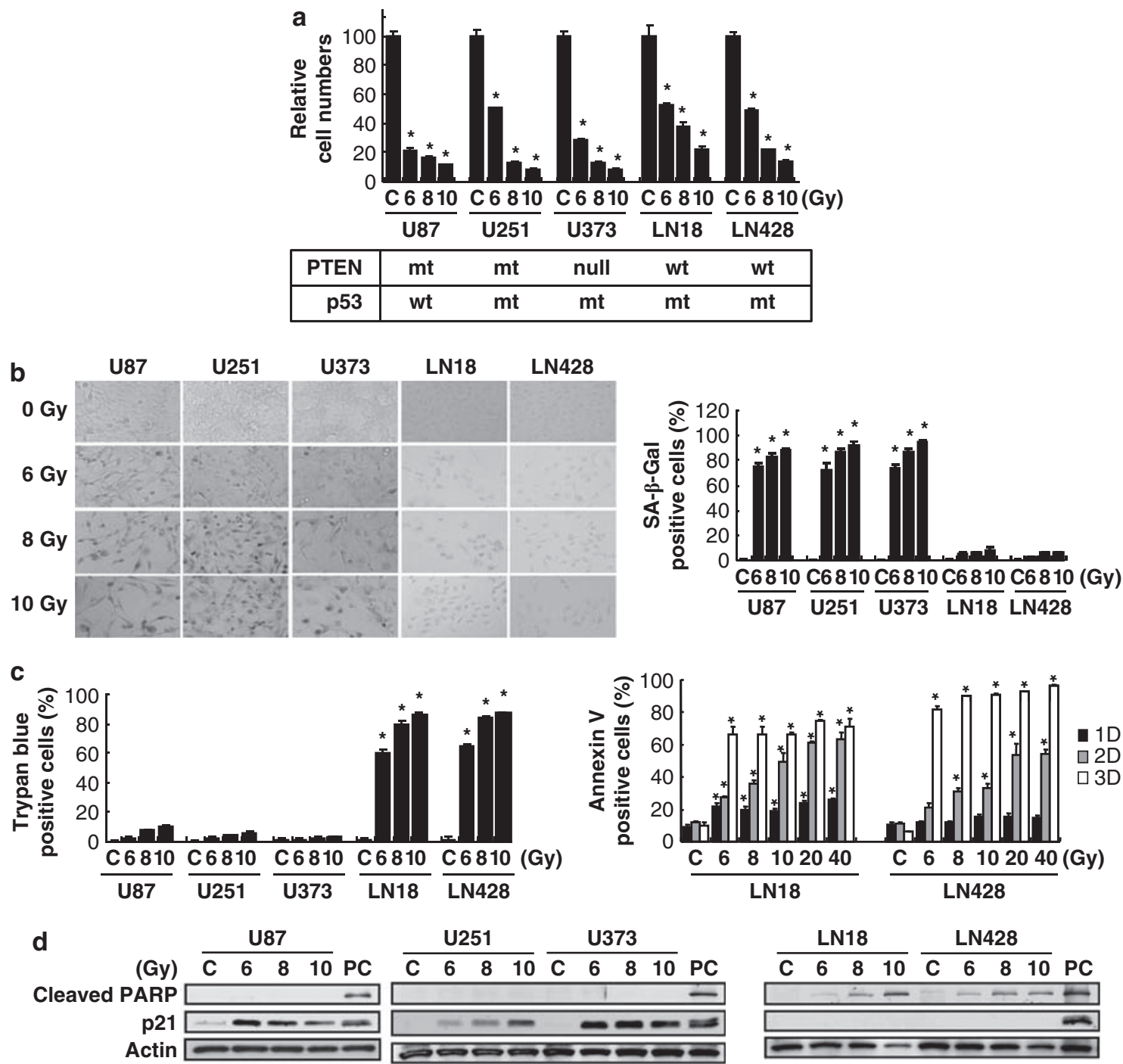

Figure 1 Phosphatase and tensin homolog status determines different cellular responses to ionizing radiation (IR). The PTEN-deficient or -proficient glioma cell lines were treated with IR at 6, 8, or 10 Gy. Relative cell numbers (a), SA- $\beta$-Gal activities (b), and trypan blue and Annexin V-positive cells (c) were quantified after 3 days or indicated days (for Annexin V positivity). Cells were photographed under phase contrast microscopy and cell numbers were counted in hemocytometer under a microscope. (d) Western blot analyses of p21 and cleaved PARP. Actin was detected as a loading control. Quantitative results are presented as mean \pm S.D. of three independent experiments. Significantly different: ${ }^{*} P<0.01$ versus control (C) or $\mathrm{C}$ at indicated days. mt, mutant; wt, wild type 

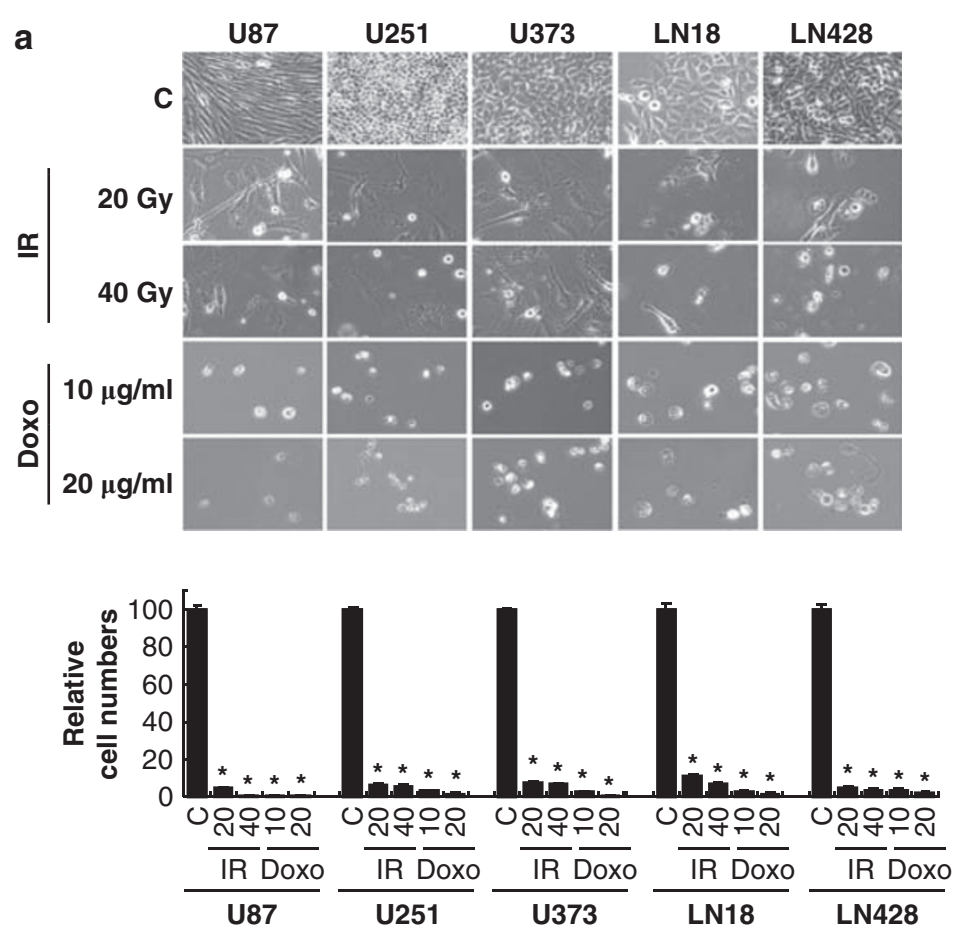

b

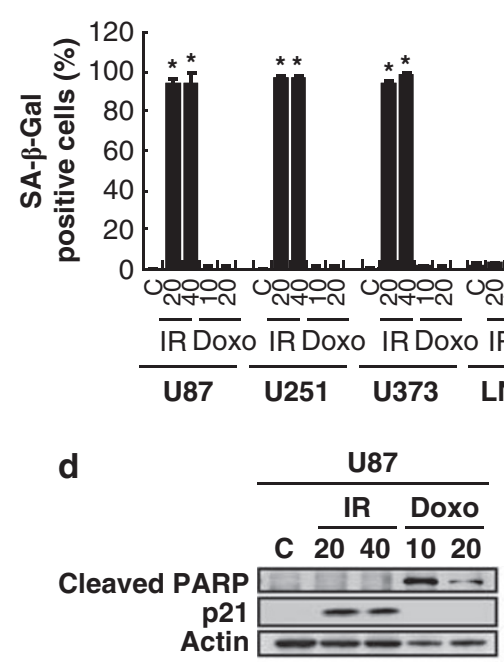

c

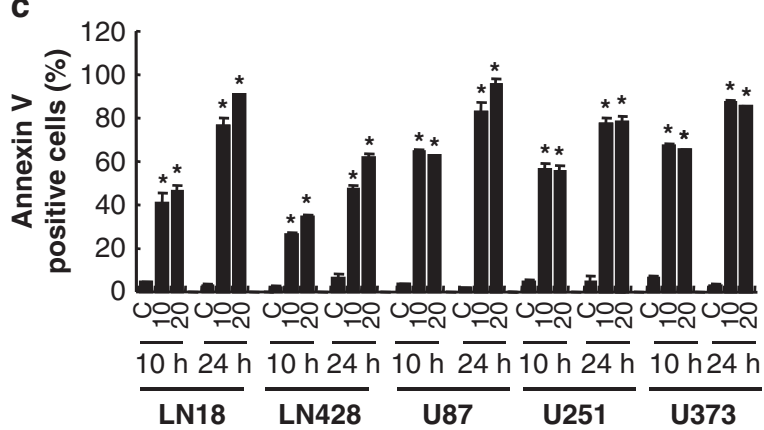

U373

$\frac{\text { U251 }}{\text { C } \frac{\text { IR }}{2040} \frac{\text { Doxo }}{1020} \text { C } \frac{\text { IR }}{2040} \frac{\text { Doxo }}{1020}}$

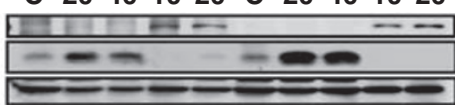

$\frac{\text { LN18 }}{\text { C } \frac{\text { IR }}{2040} \frac{\text { Doxo }}{1020} \text { C } \frac{\text { IR }}{2040} \frac{\text { Doxo }}{1020}}$

\begin{tabular}{l}
\hline$-\infty-\infty-\infty$ \\
\hline$+-\infty-\infty$
\end{tabular}

Figure 2 Phosphatase and tensin homolog (PTEN)-deficient glioma cells activate the apoptotic pathway after treatment with doxorubicin, but enter premature senescence after ionizing radiation (IR) exposure. The PTEN-deficient or -proficient glioma cell lines were treated with 20 or 40 Gy of IR, and 10 or $20 \mu \mathrm{g} / \mathrm{ml}$ of doxorubicin. Cellular morphologies (upper panel) and relative cell numbers (lower panel) (a), SA- $\beta$-Gal activities (b), and Annexin V-positive cells (c) were quantified after 3 days or 10 and $24 \mathrm{~h}$ (for Annexin V positivity). Cells were photographed under phase contrast microscopy and cell numbers were counted in hemocytometer under a microscope. (d) Western blot analyses of p21 and cleaved PARP. Actin was detected as a loading control. Each bar in the graphs indicates mean \pm S.D. of three independent experiments. Significantly different: ${ }^{*} P<0.01$ versus $C$

(Figure 1d). Caspase-3/7 activity and pro-caspase 3 cleavage were also increased in dose- and time-dependent manners to exposure to IR in LN18 and LN428 cells (Supplementary Figure S1). These data suggest that decreased cell numbers after exposure to IR are due to premature senescence in PTEN-deficient cells, and due to apoptosis in PTEN-proficient cells, regardless of p53 status. In PTEN-deficient PC-3 and PTEN-proficient DU145 prostate cancer cells, we observed cellular responses to IR that were very similar to those in glioma (Supplementary Figure S2).
PTEN-deficient gliomas adopt different final cell fates depending on stimulus type. As IR induced senescence in PTEN-deficient cells and apoptosis in PTEN-proficient cells, we next tested the effect of higher doses of IR and treatment with the genotoxic drug doxorubicin on the same cell types. When treated with 20 or $40 \mathrm{~Gy}$ of IR or with 10 or $20 \mu \mathrm{g} / \mathrm{ml}$ doxorubicin, all glioma cell numbers decreased, as compared with proliferating control cells (Figure 2a). As described before, PTEN-deficient U87, U251, and U373 cells were positive for SA- $\beta$-Gal staining after 20 or $40 \mathrm{~Gy}$ of IR 
exposure, indicating that they underwent senescence (Figure 2b). However, doxorubicin treatment induced apoptosis in PTEN-deficient cells, as evidenced by Annexin $\checkmark$ positivity and PARP cleavage (Figure $2 c$ and $d$ ), whereas PTEN-proficient LN18 and LN428 cells underwent apoptosis, regardless of stimulus type (Figure $2 a-d$ ). Levels of p21 increased after administration of high doses of IR, but not in doxorubicin-treated U87, U251, and U373 cells, and neither treatment induced p21 expression in LN18 nor LN428 cells (Figure 2d). We could not detect expression of other cell cycle regulatory proteins such as p16 and p14 (data not shown), consistent with prior data showing they are deleted in all glioma cell lines used in this study except U373. ${ }^{12}$ Cleaved PARP was observed in doxorubicin-treated U87, U251, and U373 cells and in LN18 and LN428 cells treated with high doses of IR or doxorubicin. Caspase-3/7 activity and pro-caspase 3 cleavage were detected in doxorubicintreated U87, U251, U373, LN18, and LN428 cells (Supplementary Figure S3). In contrast with the response to IR, these data demonstrate that PTEN-deficient cells underwent apoptosis, not senescence, as a result of doxorubicin treatment. This indicates that PTEN-deficient cells could activate either apoptosis or senescence pathways, depending on the type of cellular stimulus. When we applied hydrogen peroxide $\left(\mathrm{H}_{2} \mathrm{O}_{2}\right)$ to U87 and $\mathrm{LN} 18$ cells to examine cellular responses to other stimuli in the presence or the absence of PTEN, LN18 cells showed dramatic increases in the number of trypan blue positive cells and PARP cleavage, and no induction of p21, as compared with U87 cells (Supplementary Figure S4).

Reactive oxygen species are essential for the induction of senescence in U87 cells, but not for apoptosis in LN18 cells. We next examined molecular changes of senescence in PTEN-deficient U87 cells, and those of apoptosis in PTEN-proficient LN18 cells, over time following IR treatment. Both cell types had an immediate reduction in cell number and in morphological changes, and as before, only U87 cells had increased SA- $\beta$-Gal staining (Figure 3a). Furthermore, the increase in apoptotic cells, which was detected using Annexin $\mathrm{V}$ assay, was evidently observed in LN18 cells (Figure 3b), as we previously observed. As shown in Figure 3c, LN18 cells exhibited dramatic increases in PTEN, mutant p53, and cleaved PARP. However, phosphoAKT (S473) and p21 induction were not detected. In contrast, U87 cells were characterized by gradual increases in phospho-AKT, wt p53, and p21 (Figure 3c). There were no detectable PARP cleavage and PTEN induction in U87 cells.

We tested for reactive oxygen species (ROS) production in U87 and LN18 cells to know whether there was difference in the levels of ROS between premature senescence and apoptosis. Reactive oxygen species increased in both cell lines, and U87 cells exhibited significantly higher intracellular ROS levels than LN18 cells (Figure 3d, left panel). As it has been known that active AKT could reduce MnSOD and catalase expression by inhibition of Forkhead box $01 / 3$ (FOXO1/3), ${ }^{5}$ and AKT activation was detected in U87 cells after IR exposure in this study, we next tested for levels of phospho-FOXO1/3, MnSOD, and catalase. We observed no effect of IR on FOXO1/3 phosphorylation or levels of MnSOD,
$\mathrm{Cu} / \mathrm{ZnSOD}$, or catalase in U87 and LN18 glioma (Figure 3d, middle panel), indicating that increased ROS levels were not attributed to FOXO1/3 phosphorylation or the decrease of antioxidant enzymes in either of the cell lines. As mitochondrial ROS are the major source of intracellular ROS, we next measured fluorescence of MitoSOX Red as a mitochondrial superoxide indicator (Figure 3d, right panel). Fluorescence intensity of MitoSOX Red was increased in both the cell lines and more significantly in U87 cells, consistent with increased intracellular ROS levels.

To verify the role of ROS in senescence or apoptosis induced by IR, we treated U87 and LN18 cells with the ROS scavenger $\mathrm{N}$-acetyl-I-cysteine (NAC) before IR exposure (Figure 4a). Whereas NAC blocked induction of senescence in U87 cells, it did not inhibit apoptotic cell death in LN18 cells (Figure $4 b-d$ ). Relative cell numbers were decreased both in IR-treated and in NAC and IR co-treated LN18 cells (Figure $4 \mathrm{~b}$ ), and the percentage of apoptotic cells and PARP cleavage were not recovered by treatment of LN18 cells with NAC (Figure 4c and d). Likewise, we observed no increase in SA- $\beta$-Gal activity and p21 induction in LN18 cells (Figure 4c and d). In contrast, pretreatment of U87 cells with NAC before exposure to IR, reduced SA- $\beta$-Gal staining, wt p53 activation, and p21 induction, which had been induced by IR exposure (Figure $4 \mathrm{c}$ and $\mathrm{d}$ ). However, AKT activation and PARP cleavage were not affected here (Figure 4d), and total cell number and percentage of apoptotic cells were not increased (Figure $4 \mathrm{~b}$ and $\mathrm{c}$ ). Together, these data suggest that ROS generation, likely through the upstream AKT and the downstream p53/p21 signaling pathways, is indispensable for the induction of senescence phenotypes, but not apoptosis, in glioma.

Wild-type PTEN expression or AKT depletion shifts premature senescence to apoptosis in IR-exposed U87 glioma. We clarified the role of PTEN/AKT in cellular senescence by transfecting either wt PTEN or AKT small interfering RNA (siRNA) into U87 cells. Overall, we observed that transfection of either wt PTEN or AKT siRNA induced apoptosis instead of senescence as responses to IR exposure. Increases in SA- $\beta$-Gal activity and intracellular ROS levels, which were because of IR exposure, were reduced by wt PTEN expression (Figure 5a, right panel, and c). Annexin V-positive cells and caspase-3/7 activity were increased, and cleaved PARP was detected instead of p21 induction (Figure $5 b$ and $d$ ). Furthermore, cells transfected with wt PTEN alone showed apoptotic morphology and PARP cleavage (Figure $5 \mathrm{a}$, middle panel, and d), and also resulted in decreased cell number and increased percentage of apoptotic cells (Figure $5 \mathrm{a}$ and $\mathrm{b}$ ). When we further examined IR response in AKT siRNA-transfected U87 cells, AKT depletion also shifted cells from premature senescence to apoptosis as a response to IR (Figure 6), as observed in wt PTEN-expressing cells (Figure 5). AKT depletion resulted in decreases in both SA- $\beta$-Gal activity and intracellular ROS levels (Figure $6 \mathrm{a}$ and $\mathrm{c}$ ). Annexin $\mathrm{V}$ positivity, PARP cleavage, and caspase-3/7 activation in Figure $6 b$ and $d$ were evidently increased because of AKT depletion, as opposed to $\mathrm{p} 21$ induction and SA- $\beta$-Gal staining. These data confirm that PTEN is critical not only for the regulation of cell 

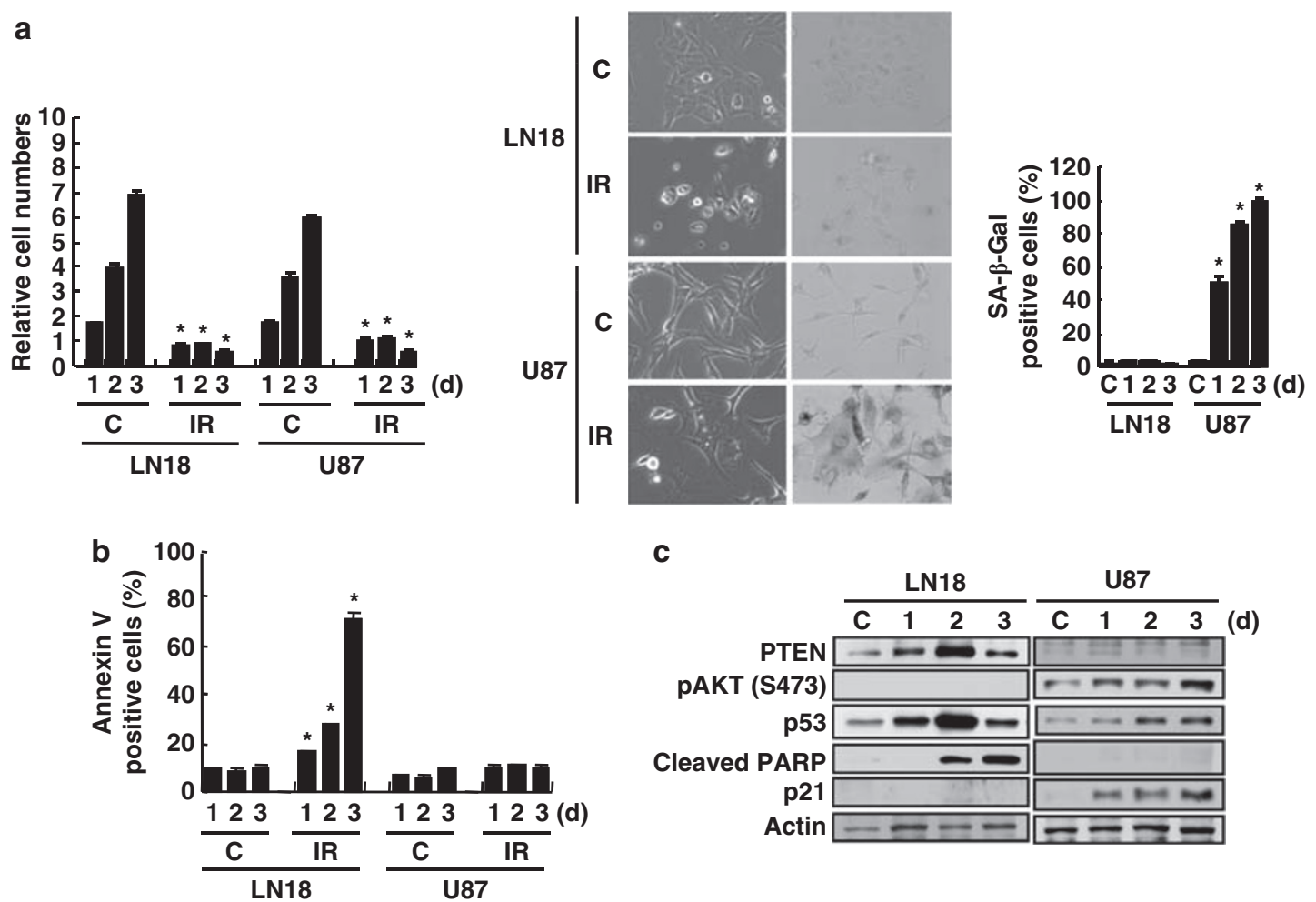

C
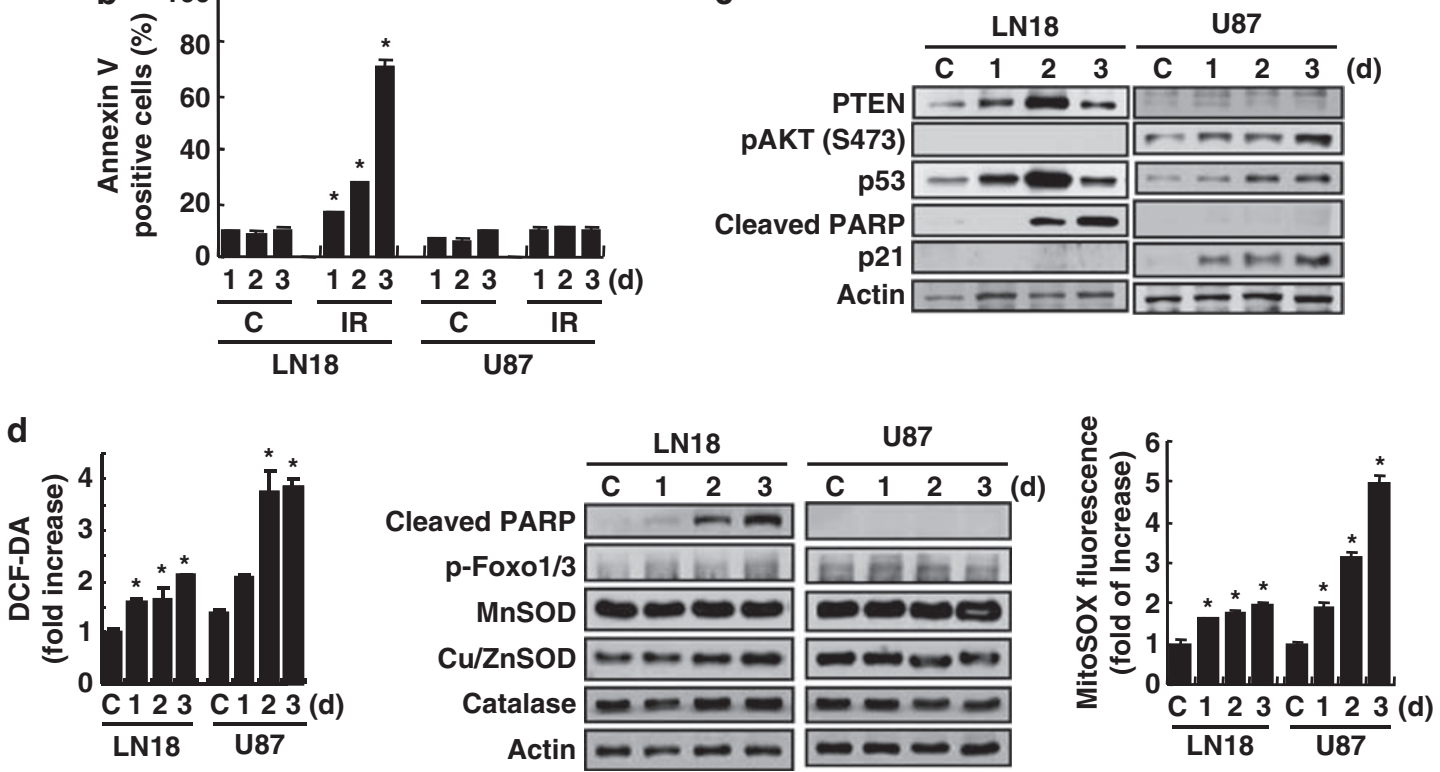

Figure 3 Cellular responses to ionizing radiation (IR) differ between PTEN-deficient U87 and PTEN-proficient LN18 cells. U87 and LN18 cells were treated with 8 Gy of IR and were analyzed as indicated at 1, 2, and 3 days post treatment. Relative cell numbers (left panel) and SA- $\beta$-Gal activities (middle and right panels) (a) and Annexin Vpositive cells $(\mathbf{b})$ were assessed at indicated time intervals. Cells were photographed under phase contrast microscopy 3 days after treatment and cell numbers were counted in hemocytometer under a microscope. (c) Western blot analyses using the antibodies indicated in the figure. Actin was detected as a loading control. (d) Intracellular ROS were measured by flow cytometry after DCF-DA staining (left panel), and mitochondrial superoxide was measured by fluorescence with MitoSOX Red (right panel). Western blot analyses were performed using the antibodies indicated in the figure (middle panel). Quantitative results are presented as mean \pm S.D. of three independent experiments. Significantly different: ${ }^{*} P<0.01$ versus $C$ at indicated days for Figure $3 a$ and $b ;{ }^{*} P<0.01$ versus $C$ for Figure $3 d$

viability under normal conditions, but also for the induction of senescence under conditions of stress, through AKT.

The p53/p21 signaling pathway is essential for the induction of IR-induced senescent phenotypes in U87 cells. We next tested if IR-induced senescence requires signaling of the p53/p21 pathway in U87 cells. We transfected siRNA specific for p53 into U87 cells expressing wt p53, and looked for senescent phenotypes (Figure 7). In p53 siRNA transfected cells before IR exposure, p21 expression was not induced (Figure 7c), and cells showed decreased SA- $\beta$-Gal activity (Figure $7 a$, right panel). When we examined whether the absence of p53 could trigger apoptosis instead of senescence, increased apoptotic cells, caspase$3 / 7$ activity, and PARP cleavage were not detected (Figure
$7 \mathrm{~b}$ and $\mathrm{c})$. Furthermore, increases in intracellular ROS and mitochondrial superoxide, which were observed in U87 cells after IR exposure, were not affected in p53 siRNAtransfected cells (Figure $7 \mathrm{~d}$, left and middle panels). Decrease in S-phase entry, which was detected using bromodeoxyuridine (BrdU) incorporation assay, was not reverted in p53 siRNA-transfected cells (Figure 7d, right panel). Although a small increase in SA- $\beta$-Gal positive cells was observed in p53-depleted cells, both SA- $\beta$-Gal activity and morphological changes were observed only after IR exposure in control siRNA-transfected cells, indicating that p53 is involved in IR-induced senescence in U87 cells. Cells transfected with p21 siRNA before IR exposure showed phenotypes similar to those transfected with p53 siRNA (Figure 8). IR-induced senescence phenotypes were not 


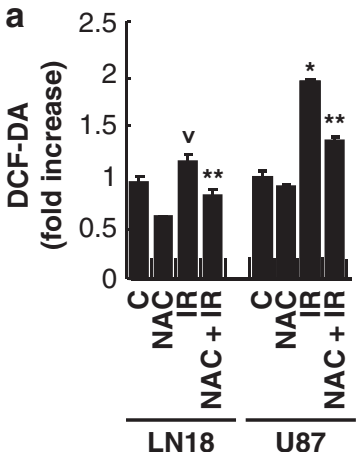

b
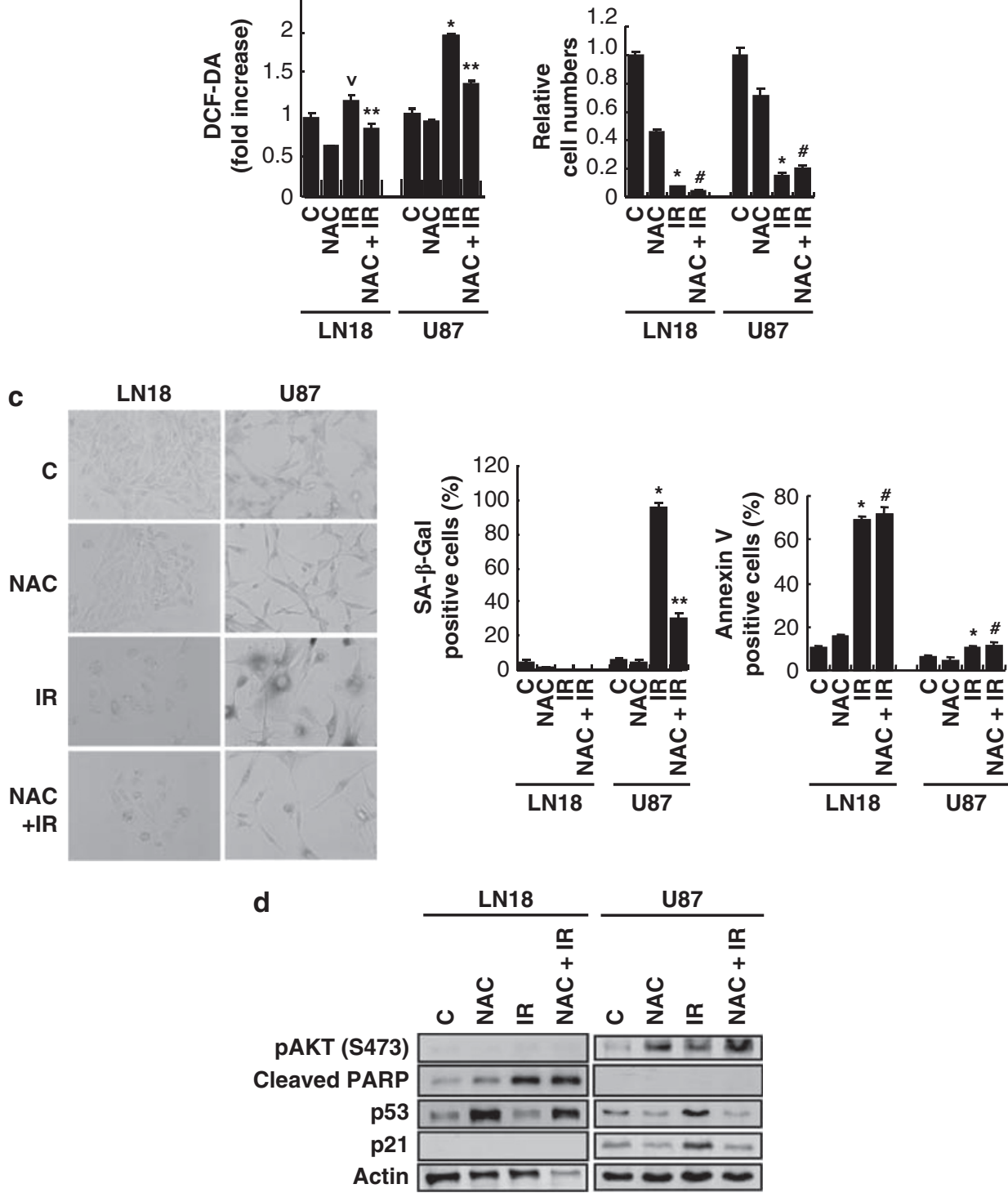

Figure 4 lonizing radiation (IR)-induced premature senescence, but not apoptosis, requires ROS accumulation. The U87 and LN18 cells were treated with 8 Gy of IR and were analyzed as indicated after 3 days in the presence or absence of $10 \mathrm{mM} \mathrm{NAC}$. (a) Intracellular ROS were measured by flow cytometry after DCF-DA staining. (b) Relative cell numbers were counted in hemocytometer under a microscope. (c) Cellular morphologies (left panel), SA- $\beta$-Gal activities (middle panel), and percentage of Annexin V-positive cells (right panel). Cellular morphologies were observed under phase contrast microscopy and cell numbers were counted in hemocytometer under a microscope. (d) Western blot analysis using the antibodies indicated in the figure. Actin was detected as a loading control. Each bar in the graphs indicates mean \pm S.D. of three independent experiments. Significantly different: ${ }^{*} P<0.01$ versus $C,{ }^{* *} P<0.01$ versus $I R$, insignificantly different: ${ }^{\vee} P>0.01$ versus $C$, ${ }^{\#} P>0.01$ versus IR

detected, such as SA- $\beta$-Gal activity (Figure $8 \mathrm{a}$, middle and right panels). Likewise, evidence of apoptosis was not detected after p21-siRNA transfection, such as increases in apoptotic cell, caspase-3/7 activity, and PARP cleavage (Figure $8 \mathrm{~b}$ and $\mathrm{c}$ ). Finally, increase in intracellular ROS level originated from mitochondria and cell cycle arrest were not returned to control levels (Figure $8 \mathrm{~d}$ ). These data suggest that depletion of either p53 or p21 directs PTEN-deficient U87 cells towards cytostatic (that is, cell cycle arrest) characteristics, rather than to apoptosis. When we analyzed cell cycle distribution, p53- or p21- depleted cells exhibited increases in aneuploid cells $(>4 N$ ) (data not shown), which has been reported as a cause of cytostasis. ${ }^{13,14}$ Altogether, the p53/p21 signaling pathway is critical for the induction of senescent phenotypes by IR exposure in PTEN-deficient U87 glioma.

As we detected increases in another cell cycle inhibitor p27 in both p53 siRNA- and p21 siRNA-transfected cells (Figure $7 \mathrm{c}$ and $8 \mathrm{c}$ ), we next co-transfected cells with p27 siRNA and either p21 siRNA or p53 siRNA to examine the role of p27 in such cytostatic state. Transfection with p27 siRNA did not affect the cytostatic status of p53 siRNA- or p21 siRNAtransfected cells (Figure $8 \mathrm{e}$ ). This could be because of additional roles of PTEN, independent of AKT, such as 


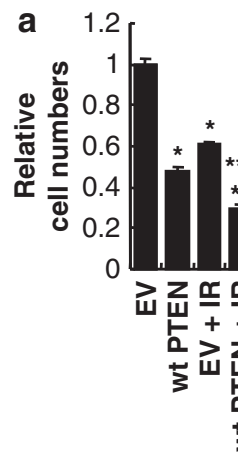

b

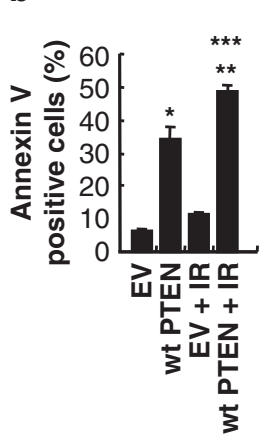

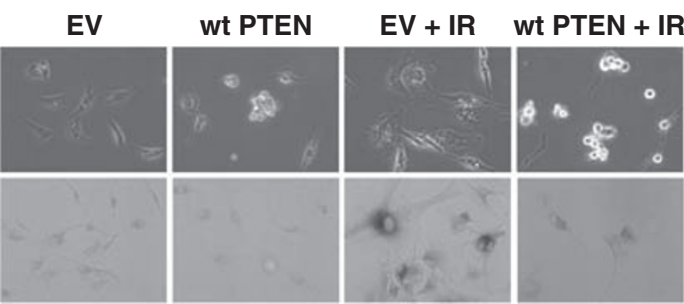

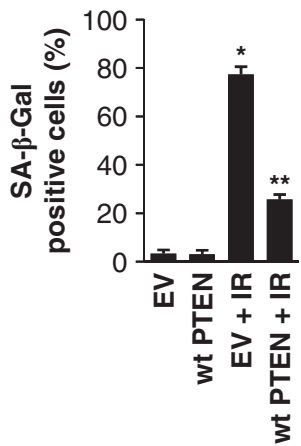

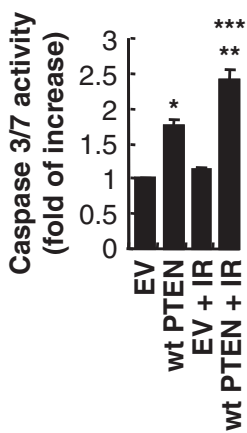

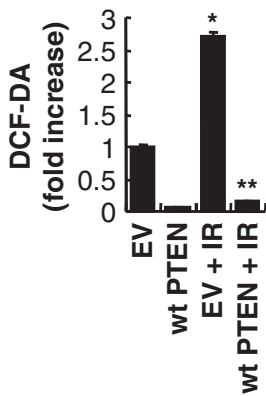

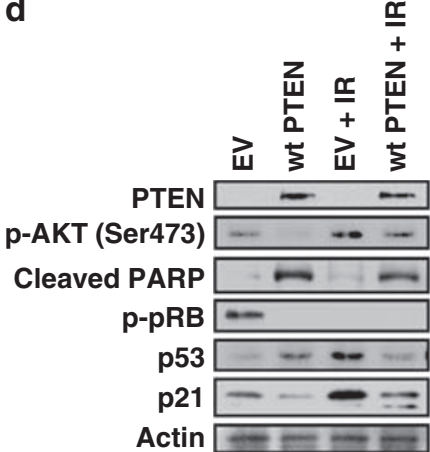

Figure 5 Wild-type (wt) PTEN expression shifts the IR response from senescence to apoptosis in U87 cells. The U87 cells were transfected with wt PTEN or empty vector (EV) followed by treatment with 8 Gy IR. (a) Relative cell numbers (left panel), cellular morphologies (middle panel), and SA- $\beta$-Gal activities (middle and right panels) were analyzed at 3 days after IR exposure. (b) Apoptotic cells were assessed by Annexin V positivity and caspase-3/7 activity at 3 days after IR exposure. (c) Intracellular ROS levels were measured by flow cytometry after DCF-DA staining at 2 days after IR exposure. (d) Western blot analysis using the antibodies indicated in the figure. Actin was detected as a loading control. Each bar in the graphs indicates mean \pm S.D. of three independent experiments. Significantly different: ${ }^{*} P<0.01$ versus EV, ${ }^{\star *} P<0.01$ versus $\mathrm{EV}+\mathrm{IR},{ }^{* * *} P<0.01$ versus wt PTEN

protein phosphatase activity. ${ }^{15}$ The mechanism regarding the cytostatic status in p53- and p21-depleted cells needs to be clarified by further experiments.

\section{Discussion}

Senescent cells are characterized by irreversible cell cycle arrest, although they remain metabolically active and acquire specific properties, such as large and flattened morphological changes, SA- $\beta$-Gal staining based on lysosomal $\beta$-galactosidase activity at $\mathrm{pH} 6.0$, resistance to apoptosis, and altered gene expression. ${ }^{16}$ More than 50 years after Hayflick and Moorehead reported replicative senescence in normal human fibroblasts, recent evidence has suggested that cellular senescence is a defense mechanism against tumorigenesis in vivo. ${ }^{17}$ In addition, as cellular senescence is abundant in premalignant neoplastic lesions and tumor regression is attributed to cellular senescence, this process is considered to have a crucial role in tumor treatment. ${ }^{18,19}$ Indeed, if cellular senescence pathways remain intact, senescence induction could compensate for the inactivation of the apoptotic pathway and could be triggered with low doses of chemotherapeutic drugs or IR. ${ }^{20}$ Therefore, it is possible that improved tumor treatments could be developed by understanding the mechanism of premature senescence. ${ }^{9}$

Gliomas are the most common malignant brain tumor in adults and are among the most lethal of all cancers. ${ }^{21,22}$
Glioblastomas contain a number of clearly defined genetic lesions, which result in disruption of critical cellular signaling pathways that regulate cell proliferation. Activation of the AKT oncogene by mutational inactivation of the PTEN tumor suppressor, or mutational activation of PI3K, is common in glioblastomas. ${ }^{23}$ As these genetic lesions regulate biological and clinical behaviors of tumor cells, the resultant disrupted signaling pathways represent attractive targets for therapy. ${ }^{24}$

The aim of this study was to elucidate whether PTEN status could alter cellular responses to IR using five different glioma cell lines. We found that PTEN-deficient U87, U373, and U252 glioma cells, which have an active AKT signaling pathway because of the absence of PTEN, entered senescence following IR exposure. However, the PTEN-proficient cell lines LN428 and LN18 underwent apoptosis under the same treatment conditions. AKT has crucial roles not only in the proliferation and survival of mammalian cells, thereby promoting tumorigenesis, but also for the induction of premature senescence. ${ }^{6,25}$ In this study, U87 cells depleted of either p53 or p21 did not develop a senescent phenotype, but instead remained cytostatic as opposed to triggering apoptosis in response to activated AKT. ${ }^{26,27}$ In addition, acute PTEN inactivation induces growth arrest through the p53-dependent cellular senescence pathway both in vitro and in vivo. ${ }^{28}$ PTEN/AKT might exert another regulatory function(s) of cell proliferation through a p53/p21-independent pathway. For example, as PTEN has recently been reported 

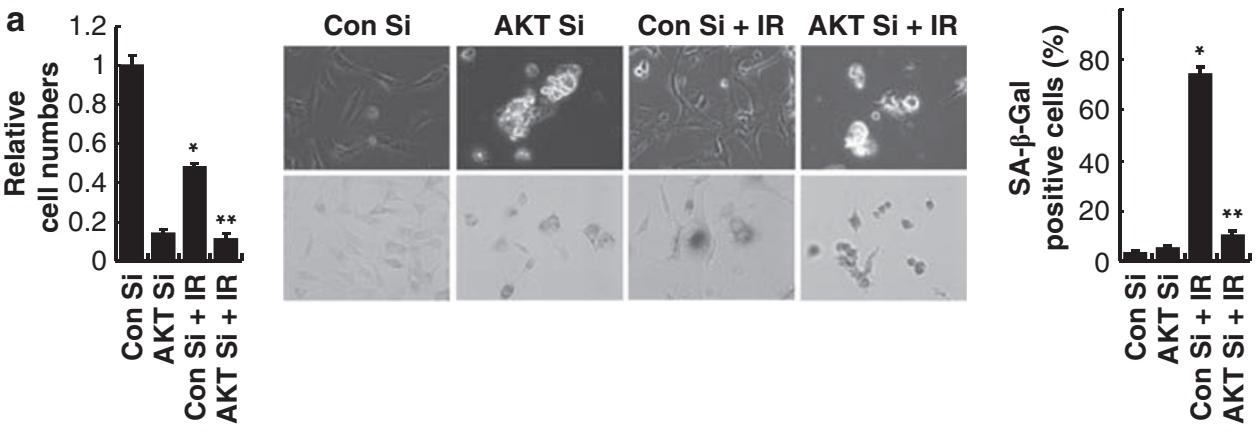

b

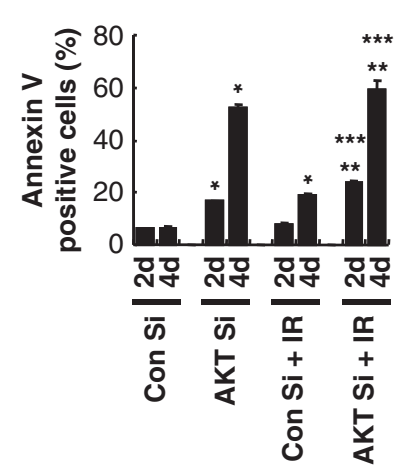

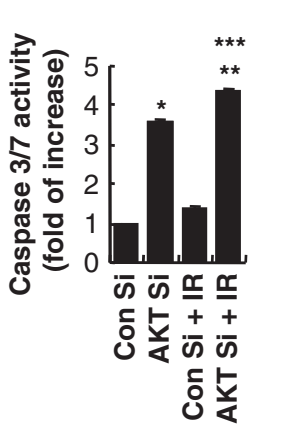

c

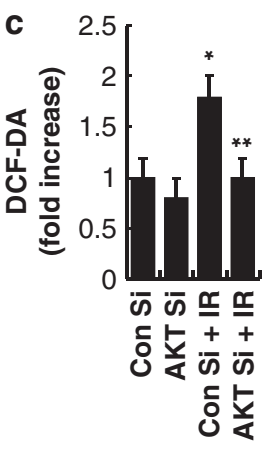

d

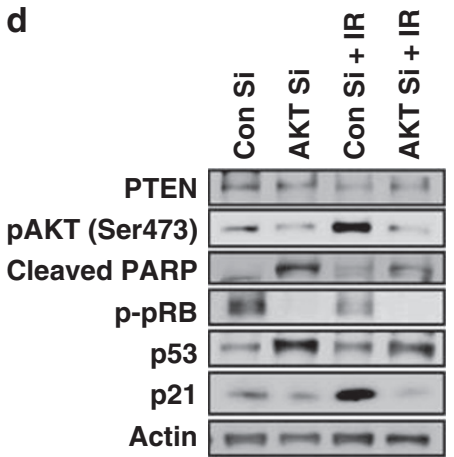

Figure 6 Depletion of AKT shifts the IR response from senescence to apoptosis in U87 cells. The U87 cells were transfected with AKT siRNA or control siRNA followed by treatment with 8 Gy IR. (a) Relative cell numbers (left panel), cellular morphologies (middle panel), and SA- $\beta$-Gal activities (middle and right panels) analyzed at 3 days after IR exposure. (b) Percentages of Annexin V-positive cells and caspase-3/7 activity. Annexin V positivity and caspase-3/7 activity were assessed 2 and 4 days or 3 days after treatment, respectively. (c) Intracellular ROS levels were measured by flow cytometry after DCF-DA staining 2 days after IR exposure. (d) Western blot analysis using the antibodies indicated in the figure. Actin was detected as a loading control. Con, control; si, siRNA. Each bar in the graphs indicates mean \pm S.D. of three independent experiments. Significantly different: ${ }^{\star} P<0.01$ versus Con si, ${ }^{\star \star} P<0.01$ versus Con si $+\mathrm{IR},{ }^{\star \star *} P<0.01$ versus AKT si

to have protein phosphatase activity in addition to lipid phosphatase activity, ${ }^{15}$ PTEN may have additional roles in tumor biology, independent of AKT activity and the senescence pathway. As we found that p27, another cell cycle inhibitor, did not have a role in the cytostatic status of p53- and p21-depleted cells, additional experiments will need to be performed before the details regarding this mechanism are understood.

Although the PI3K/AKT pathway is involved in both premature senescence and cell proliferation, the specific mechanism related to this process is not fully understood. Here, we observed that different responses by glioma cell lines are dependent on PTEN status, and that PTEN has a critical role in switching cell fate between senescence and apoptosis after IR exposure. We also found that depletion of AKT or scavenging of ROS prevented IR-induced senescence in PTEN-deficient glioma. These data suggest that the effect of PTEN on cellular senescence is likely mediated by increased intracellular ROS by AKT activation. Since the discovery that $\mathrm{H}_{2} \mathrm{O}_{2}$ can induce apoptosis, many reports have demonstrated the importance of ROS in the apoptotic pathway in various cell systems. ${ }^{29,30}$ However, it has also been reported that significant levels of ROS are produced in senescing cells, but not in apoptotic cells. ${ }^{31}$ Similar studies have shown that ROS are also produced in cells undergoing apoptotic-like cell death, but that the produced ROS do not cause apoptosis. ${ }^{32}$ In this study, we observed that ROS were not required to induce apoptosis in PTEN-proficient glioma.
Recent reports suggested that constitutive activation of AKT promotes senescence-like arrest of cell growth through inhibition of the transcription factor FOXO1/3. , $^{63}$ Inhibition of FOXO1/3 increases intracellular ROS through the regulation of antioxidant enzymes levels, such as MnSOD, sestrin, and catalase, and many experiments have shown that ROS have a critical role in determining life span and cellular senescence in mammalian cells. ${ }^{5}$ In this study, ROS levels were elevated during IR-induced senescence, and the ROS scavenger NAC reverted IR-induced senescence phenotypes, including p53 activation, p21 induction, and SA- $\beta$-Gal activity. However, increases in intracellular ROS levels were not attributed to the phosphorylation of FOXO1/3 through AKT activation.

Radiotherapy (RT) is one of major regimes for cancer therapy, and research in radiation biology is currently focused on identifying more effective means to increase RT efficacy. ${ }^{34}$ It is accepted that apoptosis has a primary role in tumor regression after $\mathrm{RT}$, and many studies have attempted to elucidate applicable mechanisms to increase cells undergoing apoptosis in RT-targeted tumors. ${ }^{35,36}$ It has been known that apoptosis is readily induced in tumors derived from hematopoietic, lymphoid, and germ cells, but some solid tumors derived from epithelial cells show resistance to apoptosis after IR exposure. ${ }^{37,38}$ Recent evidence has shown that IR prematurely induces senescent phenotypes, instead of apoptosis, before the Hayflick limit, which is known as stressinduced premature senescence. We also previously reported 


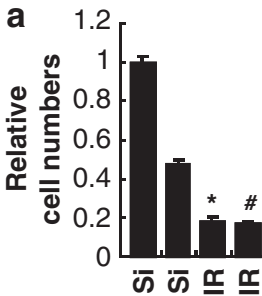
ठํํำ

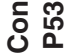

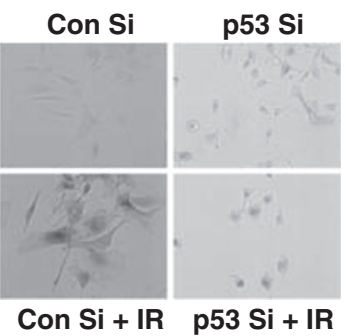

Con Si + IR p53 Si + IR

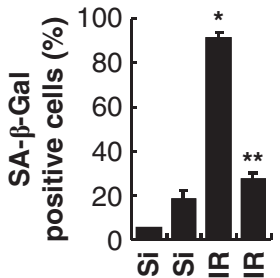

о

ठํ ณ

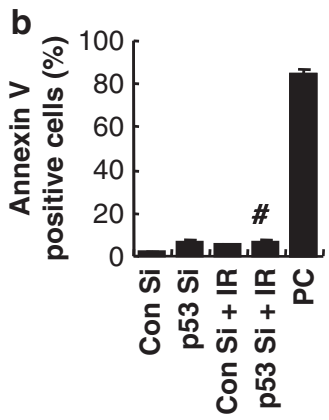

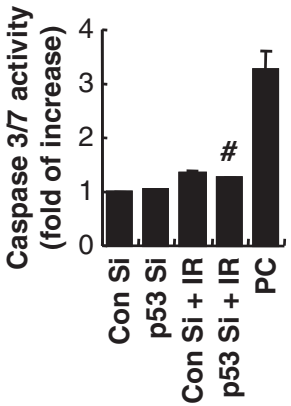

C

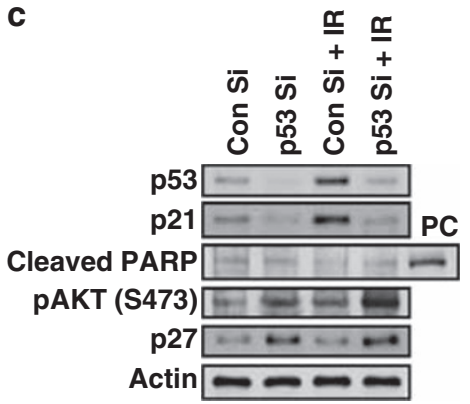

d
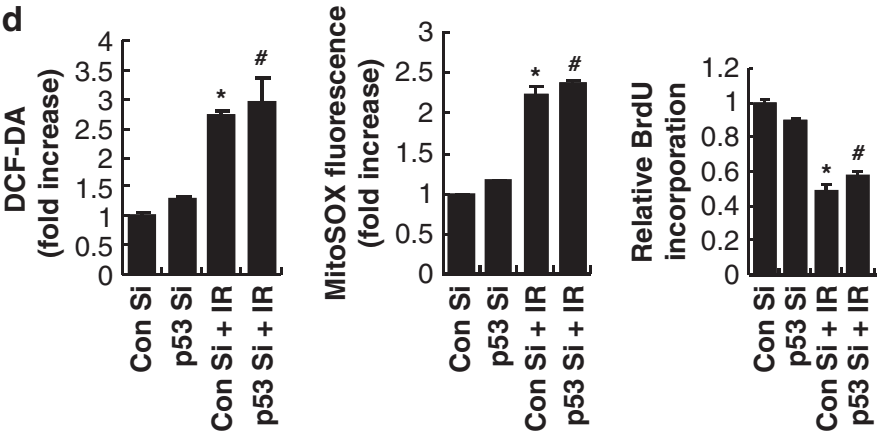

Figure 7 Knockdown of p53 inhibits IR-induced premature senescence. The U87 cells were transfected with p53 siRNA or control siRNA before 8 Gy of IR exposure. (a) Relative cell numbers (left panel) and SA- $\beta$-Gal activities (middle and right panels) were quantified 4 days after IR exposure. (b) Percentage of Annexin V-positive cells and caspase-3/7 activity analyzed 3 days after treatment. (c) Western blot analysis using the antibodies indicated in the figure. Actin was detected as a loading control. (d) Intracellular ROS levels (left panel), mitochondrial superoxide levels (middle panel), and relative BrdU incorporation (right panel) were measured 3 days after IR exposure. Intracellular ROS were measured by flow cytometry after DCF-DA staining, and mitochondrial superoxide was measured by fluorescence with MitoSOX Red. Con, control; si, siRNA; PC, positive control (U87 cells treated with doxorubicin $10 \mu \mathrm{g} / \mathrm{ml}$ for $24 \mathrm{~h}$ ). Quantitative results are presented as mean \pm S.D. of three independent experiments. Significantly different: ${ }^{*} P<0.01$ versus Con $\mathrm{si}$, ${ }^{\star} P<0.01$ versus Con $\mathrm{si}+\mathrm{IR}$, insignificantly different (?): ${ }^{\#} P>0.01$ versus Con si $+\mathrm{IR}$

that IR induces premature senescence in breast, colon, and lung carcinoma, and in tumor tissue of xenografted mice. ${ }^{20,39}$ Therefore, understanding premature senescence mechanisms could provide helpful information for improving the efficacy of RT.

In this study, we describe for the first time a detailed molecular mechanism of IR-induced senescence in PTENdeficient glioma. Premature senescence has a compensatory role in apoptosis in the absence of the tumor suppressor PTEN through the AKT/ROS/p53/p21 pathway, whereas PTEN proficiency sensitizes glioma cells to apoptosis, independently of p53 and ROS (Figure 8f). Conclusively, our results affirm that $\mathrm{RT}$-induced premature senescence could be an alternative fail-safe treatment option in apoptosisresistant glioma cells, and we expect our findings may aid in the understanding of the clinical significance of premature senescence in PTEN-deficient tumor cells.

\section{Materials and Methods}

Materials. LN18 and U87 glioma cell lines were purchased from American Type Culture Collections (Manassas, VA, USA) and U251, U373, and LN428 cell lines were obtained from Prof. S-J Lee in Hanyang University (Seoul, Korea). These cell lines were cultured in Dulbecco's modified Eagle's medium (HyClone, Logan, UT, USA) supplemented with $10 \%$ fetal bovine serum (WeIGENE, Daegu, Korea) and $25 \mathrm{U} / \mathrm{ml}$ penicillin/streptomycin. The p53 antibody was purchased from Novocastra Inc. (Newcastle, UK). Phospho-pRB, phspho-AKT, and PARP antibodies were obtained from Cell Signaling Inc. (Danvers, MA, USA). Antibodies for p27, p21, $\beta$-actin, and HRP-conjugated goat anti-mouse were purchased from Santa Cruz Biotechnology (Santa Cruz, CA, USA). Hoseradish peroxidase-conjugated antirabbit and anti-mouse antibodies were obtained from Amersham Inc. (Uppsala, Sweden). Wild-type PTEN construct was a generous gift by Dr. Y-E Whang in 
a

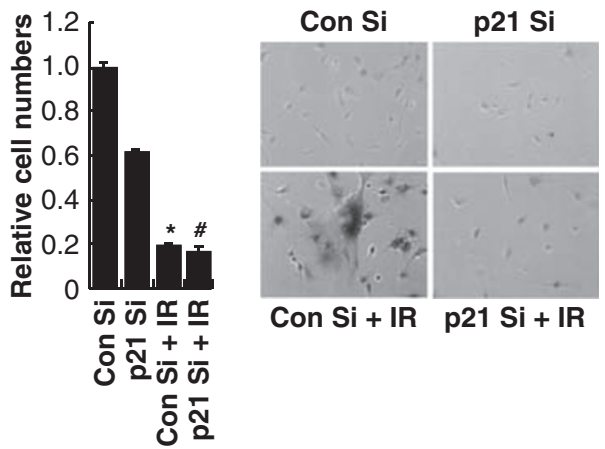

C

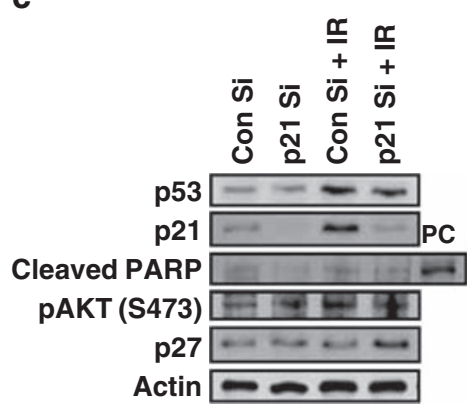

b

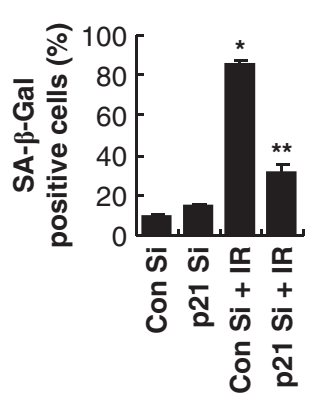

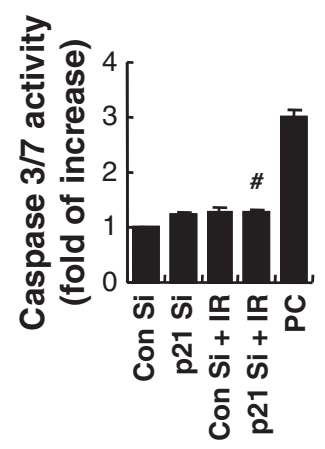

d

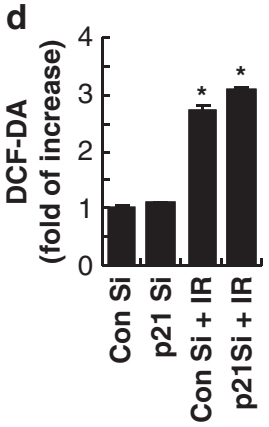

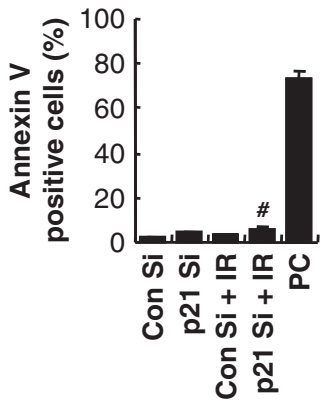

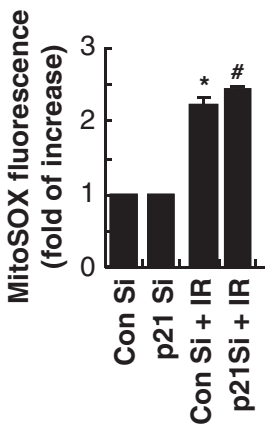

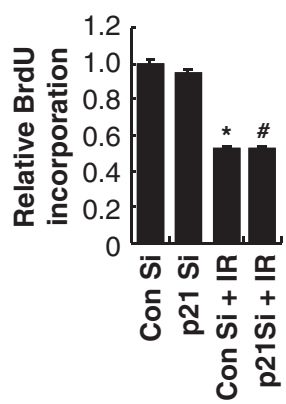
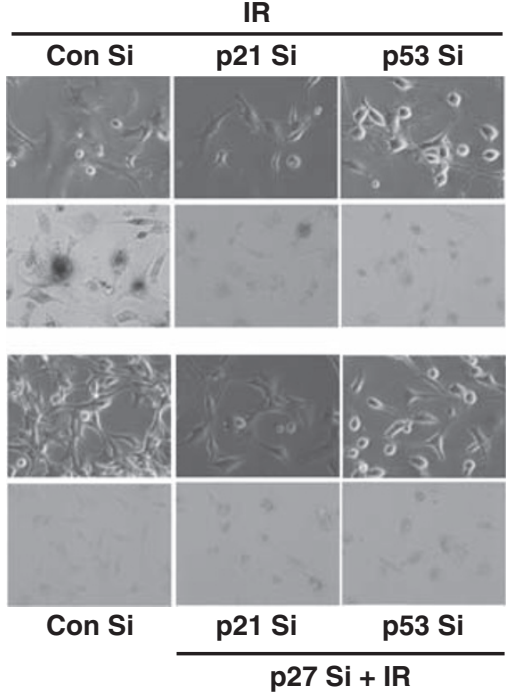

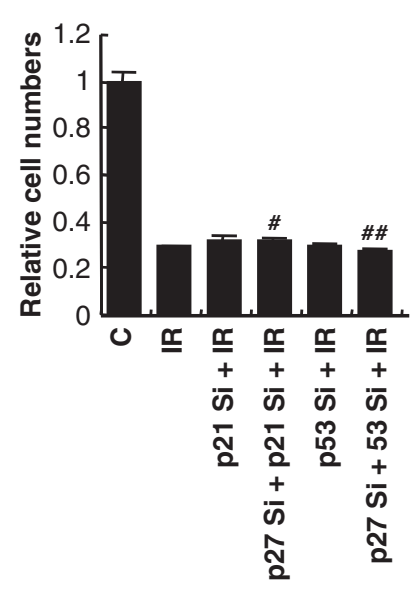

f

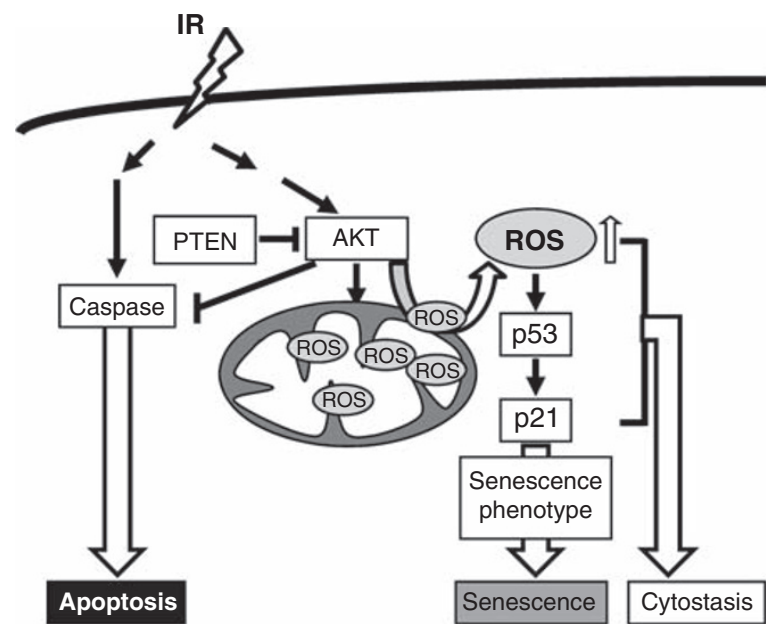

Figure 8 Knockdown of p21 inhibits IR-induced premature senescence. The U87 cells were transfected with p21 siRNA or control siRNA before 8 Gy of IR exposure. (a) Relative cell numbers (left panel) and SA- $\beta$-Gal activities (middle and right panels) were quantified 4 days after IR exposure. (b) Percentages of Annexin V-positive cells and caspase-3/7 activity analyzed at 3 days after IR exposure. (c) Western blot analysis using the antibodies indicated in the figure. Actin was detected as a loading control. (d) Intracellular ROS levels (left panel), mitochondrial superoxide levels (middle panel), and relative BrdU incorporation (right panel) were measured 3 days after IR exposure. Intracellular ROS were measured by flow cytometry after DCF-DA staining, and mitochondrial superoxide was measured by fluorescence with MitoSOX Red. (e) Cellular morphologies and SA- $\beta$-Gal activities (left panel) and relative cell numbers (right panel) in U87 cells, which were transfected with p53 siRNA or p21 siRNA in the presence or absence of p27 siRNA followed by treatment with 8 Gy IR. Cellular morphologies were observed under phase contrast microscopy and cell numbers were counted in hemocytometer under a microscope at 3 days after IR exposure. Con, control; si, siRNA; PC, positive control (U87 cells treated with doxorubicin $10 \mu \mathrm{g} / \mathrm{ml}$ for $24 \mathrm{~h}$ ). (f) A model illustrating the role of PTEN in switching the cell fate between premature senescence and apoptosis by IR. Quantitative results are presented as mean \pm S.D. of three independent experiments. Significantly different: ${ }^{*} P<0.01$ versus Con si, insignificantly different: ${ }^{\#} P>0.01$ versus Con si + IR for Figure 8 a-d, ${ }^{\#} P>0.01$ versus $p 21$ $\mathrm{si}+\mathrm{IR}$ for Figure $8 \mathrm{e},{ }^{\#} P>0.01$ versus $\mathrm{p53} \mathrm{si}+\mathrm{IR}$ for Figure $8 \mathrm{e}$

University of North Carolina School of Medicine (Chapel Hill, NC, USA). Small interfering RNA duplexes against AKT, p21, and p27 were purchased from Bioneer Inc. (Daejeon, Korea), and p53 siRNA was purchased from Santa Cruz
Biotechnology. $\mathrm{N}$-acetyl-I-cysteine and doxorubicin were obtained from Calbiochem (San Diego, CA, USA), and 2', $7^{\prime}$-dichlorofluorescin diacetate was purchased from Invitrogen (Carlsbad, CA, USA). 
Irradiation. For irradiation, cells were exposed to $\gamma$-ray with a ${ }^{137} \mathrm{Cs} \gamma$-ray source (Atomic Energy of Canada Ltd.) at a dose rate of $3.2 \mathrm{~Gy} / \mathrm{min}$.

Trypan blue staining. Trypan blue solution $(0.4 \%)$ was added to the cell suspension at a 1:5 ratio, and incubated for $5 \mathrm{~min}$ at room temperature. Cells excluding the stain were counted in a hemocytometer under a microscope as viable cells.

Measurement of caspase-3/7 activity. Caspase-3/7 activity was measured by using the Apo-one Homogenous Caspase-3/7 Assay kit (Promega, Madison, WI, USA) following the protocol provided by the manufacturer. In brief, cells were trypsinized and mixed with the same volume of the Apo-one Homogenous Caspase-3/7 reagent. After incubation at room temperature for $3 \mathrm{~h}$, caspase-3/7 activities were measured from the fluorescence at the excitation wavelength of $485 \mathrm{~nm}$ and the emission wavelength of $535 \mathrm{~nm}$ using the Fluoroskan Ascent Synergy (Biotex Instrument, Highland Park, IL, USA).

Annexin V staining. Apoptosis-mediated cell death was examined using a FITC-Annexin V apoptosis detection kit (BD Biosciences, San Diego, CA, USA) according to the manufacturer's instructions. Briefly, the harvested $1 \times 10^{5}$ cells were washed with phosphate-buffered saline (PBS) and resuspended in $100 \mu \mathrm{l}$ binding buffer. Then, $5 \mu$ l of FITC Annexin $V$ and $5 \mu$ l of propodium iodide (PI) were added. Flow cytometric analysis was performed after incubation for $15 \mathrm{~min}$ in the dark. Data acquisition and analysis were performed in a flow cytometer (FACSCaliber: Becton-Dickinson, Franklin Lakes, NJ, USA) using CellQuest software (BD Biosciences, San Jose, CA, USA).

Bromodeoxyuridine incorporation assay. Bromodeoxyuridine incorporation was assessed using a colorimetric enzyme-linked immunosorbent assay kit (Roche Applied Science, Indianapolis, IN, USA) according to the manufacturer's instructions.

Propidium iodide staining. Cells were fixed in $70 \%$ ethanol and washed in PBS and then stained with $0.5 \mathrm{ml}$ of PBS containing $1 \mathrm{mg} / \mathrm{ml} \mathrm{RNase}$ and $10 \mu \mathrm{g} / \mathrm{ml} \mathrm{PI}$ for $20 \mathrm{~min}$ in the dark at room temperature. The data were analyzed using Multicycle software (Phoenix Flow Systems, San Diego, CA, USA).

Measurements of ROS and mitochondrial superoxide. ROS and mitochondrial superoxide were measured using the protocol described in Lee et al..$^{39}$

Western blot analysis. For western blot analysis, we followed the protocol described in Byun et al. ${ }^{20}$

Transfection. Transfection was carried out using Lipofectamine 2000 reagent (Invitrogen) according to the manufacturer's instructions.

RNA interference. Cells were transfected with $100 \mathrm{nM}$ siRNA duplexes by using Lipofectamine RNAiMAX according to manufacturer's protocol (Invitrogen). Nonspecific siRNA (AGGUAGUGUAAUCGCCUUGUU; Bioneer, Inc., Daejeon, Korea) was used as a control for comparison.

Senescence associated $\beta$-galactosidase staining. We followed the protocol described in Dimri et al. ${ }^{40}$

Statistical analysis. Differences between various experimental groups were calculated using either Student's $t$-test or analysis of variance test for multiple comparisons. $P$-values of $<0.01$ were considered significant.

\section{Conflict of interest}

The authors declare no conflict of interest.

Acknowledgements. This work was supported by the Nuclear Research \& Development Program and Radiological Translational Research Program (RTR1003) of the Korea Science and Engineering Foundation grant funded by the Korean government (MEST).

1. Hirose Y, Berger MS, Pieper RO. Abrogation of the Chk1-mediated G(2) checkpoint pathway potentiates temozolomide-induced toxicity in a p53-independent manner in human glioblastoma cells. Cancer Res 2001; 61: 5843-5849.
2. Ohgaki H, Dessen $P$, Jourde B, Horstmann S, Nishikawa T, Di Patre PL et al. Genetic pathways to glioblastoma: a population-based study. Cancer Res 2004; 64: 6892-6899.

3. Bleeker FE, Lamba S, Zanon C, van Tilborg AA, Leenstra S, Troost D et al. Absence of AKT1 mutations in glioblastoma. PLoS One 2009; 4: e5638.

4. Keniry M, Parsons R. The role of PTEN signaling perturbations in cancer and in targeted therapy. Oncogene 2008; 27: 5477-5485.

5. Nogueira V, Park Y, Chen CC, Xu PZ, Chen ML, Tonic I et al. Akt determines replicative senescence and oxidative or oncogenic premature senescence and sensitizes cells to oxidative apoptosis. Cancer Cell 2008; 14: 458-470.

6. Miyauchi H, Minamino T, Tateno K, Kunieda T, Toko H, Komuro I. Akt negatively regulates the in vitro lifespan of human endothelial cells via a p53/p21-dependent pathway. EMBO J 2004; 23: 212-220.

7. Braig M, Schmitt CA. Oncogene-induced senescence: putting the brakes on tumor development. Cancer Res 2006; 66: 2881-2884.

8. Collado M, Serrano M. Senescence in tumours: evidence from mice and humans. Nat Rev Cancer 2010; 10: 51-57.

9. Roninson IB. Tumor cell senescence in cancer treatment. Cancer Res 2003; 63: 2705-2715.

10. Hornsby PJ. Senescence as an anticancer mechanism. J Clin Oncol. 2007; 25 1852-1857.

11. Wu CH, van Riggelen J, Yetil A, Fan AC, Bachireddy P, Felsher DW. Cellular senescence is an important mechanism of tumor regression upon c-Myc inactivation. Proc Natl Acad Sci USA 2007; 104: 13028-13033.

12. Ishii N, Maier D, Merlo A, Tada M, Sawamura Y, Diserens AC et al. Frequent co-alterations of TP53, p16/CDKN2A, p14ARF, PTEN tumor suppressor genes in human glioma cell lines. Brain Pathol 1999; 9: 469-479.

13. Dalton WB, Yu B, Yang VW. p53 suppresses structural chromosome instability after mitotic arrest in human cells. Oncogene 2010; 29: 1929-1940.

14. Tao $Y$, Leteur $C$, Yang $C$, Zhang $P$, Castedo $M$, Pierré $A$ et al. Radiosensitization by Chir-124, a selective CHK1 inhibitor. Cell Cycle 2009; 8: 1196-1205.

15. Cai XM, Tao BB, Wang LY, Liang YL, Jin JW, Yang Y et al. Protein phosphatase activity of PTEN inhibited the invasion of glioma cells with epidermal growth factor receptor mutation type III expression. Int J Cancer 2005; 117: 905-912.

16. Schmitt CA. Cellular senescence and cancer treatment. Biochim Biophys Acta 2007; 1775: $5-20$.

17. Xue W, Zender L, Miething C, Dickins RA, Hernando E, Krizhanovsky V et al. Senescence and tumour clearance is triggered by p53 restoration in murine liver carcinomas. Nature 2007; 445: 656-660.

18. Wu CH, van Riggelen J, Yetil A, Fan AC, Bachireddy P, Felsher DW. Cellular senescence is an important mechanism of tumor regression upon c-Myc inactivation. Proc Natl Acad Sci USA 2007; 104: 13028-13033.

19. Ventura A, Kirsch DG, McLaughlin ME, Tuveson DA, Grimm J, Lintault L et al. Restoration of p53 function leads to tumour regression in vivo. Nature 2007; 445 661-665.

20. Byun HO, Han NK, Lee HJ, Kim KB, Ko YG, Yoon G et al. Cathepsin D and eukaryotic translation elongation factor 1 as promising markers of cellular senescence. Cancer Res 2009; 69: 4638-4647.

21. Mischel PS, Cloughesy TF. Targeted molecular therapy of GBM. Brain Pathol 2003; 13 52-61.

22. Wang Y, Zhu S, Cloughesy TF, Liau LM, Mischel PS. p53 disruption profoundly alters the response of human glioblastoma cells to DNA topoisomerase I inhibition. Oncogene 2004; 23: $1283-1290$.

23. Solomon DA, Kim JS, Jenkins S, Ressom H, Huang M, Coppa N et al. Identification of p18 INK4C as a tumor suppressor gene in glioblastoma multiforme. Cancer Res 2008; 68 2564-2569.

24. Mischel PS, Shai R, Shi T, Horvath S, Lu KV, Choe G et al. Identification of molecular subtypes of glioblastoma by gene expression profiling. Oncogene 2003; 22: 2361-2373.

25. Minamino T, Miyauchi H, Tateno K, Kunieda T, Komuro I. Akt-induced cellular senescence: implication for human disease. Cell Cycle 2004; 3: 449-451.

26. Shiohara M, Gombart AF, Berman JD, Koike K, Komiyama A, Koeffler HP. Cytostatic effect of TNF $\alpha$ on cancer cells is independent of p21WAF1. Oncogene 1997; 15: 1605-1609.

27. Sagulenko V, Muth D, Sagulenko E, Paffhausen T, Schwab M, Westermann F. Cathepsin $D$ protects human neuroblastoma cells from doxorubicin-induced cell death Carcinogenesis 2008; 29: 1869-1877.

28. Chen Z, Trotman LC, Shaffer D, Lin HK, Dotan ZA, Niki M et al. Crucial role of p53-dependent cellular senescence in suppression of Pten-deficient tumorigenesis. Nature 2005; 436: 725-730.

29. Das M, Mukherjee SB, Shaha C. Hydrogen peroxide induces apoptosis-like death in Leishmania donovani promastigotes. J Cell Sci 2001; 114: 2461-2469.

30. Quillet-Mary A, Jaffrézou JP, Mansat V, Bordier C, Naval J, Laurent G. Implication of mitochondrial hydrogen peroxide generation in ceramide-induced apoptosis. J Biol Chem 1997; 272: 21388-21395

31. Song YS, Lee BY, Hwang ES. Distinct ROS and biochemical profiles in cells undergoing DNA damage-induced senescence and apoptosis. Mech Ageing Dev 2005; 126: 580-590.

32. Derouet-Hümbert E, Drãgan CA, Hakki T, Bureik M. ROS production by adrenodoxin does not cause apoptosis in fission yeast. Apoptosis 2007; 12: 2135-2142. 
33. Sun Y, St Clair DK, Xu Y, Crooks PA, St Clair WH. A NADPH oxidase-dependent redox signaling pathway mediates the selective radiosensitization effect of parthenolide in prostate cancer cells. Cancer Res 2010; 70: 2880-2890.

34. Suzuki M, Boothman DA. Stress-induced premature senescence (SIPS): influence of SIPS on radiotherapy. J Radiat Res 2008; 49: 105-112.

35. Dewey WC, Ling CC, Meyn RE. Radiation-induced apoptosis: relevance to radiotherapy. Int J Radiat Oncol Biol Phys 1995; 33: 781-796.

36. Ross GM. Induction of cell death by radiotherapy. Endocr Relat Cancer 1999; 6 : $41-44$.
37. Hendry $\mathrm{JH}$, Potten CS, Merritt A. Apoptosis induced by high- and low-LET radiations. Radiat Environ Biophys 1995; 34: 59-62.

38. Nomura T, Kinuta M, Hongyo T, Nakajima H, Hatanaka T. Programmed cell death in whole body and organ systems by low dose radiation. J Radiat Res 1992; 33: 109-123.

39. Lee JJ, Lee JH, Ko YG, Hong SI, Lee JS. Prevention of premature senescence requires JNK regulation of $\mathrm{Bcl}-2$ and reactive oxygen species. Oncogene 2010; 29: 561-575.

40. Dimri GP, Lee X, Basile G, Acosta M, Scott G, Roskelley C et al. A biomarker that identifies senescent human cells in culture and in aging skin in vivo. Proc Natl Acad Sci USA 1995; 92: 9363-9367.

Supplementary Information accompanies the paper on Cell Death and Differentiation website (http://www.nature.com/cdd) 